\title{
The effect of surface properties of EVAL membranes on the inflammatory response
}

\author{
Chia-Tung Shun ${ }^{\mathrm{a}}$, Tai-Horng Young ${ }^{\mathrm{b}, *}$ \\ ${ }^{a}$ Department of Pathology, College of Medicine, National Taiwan University Hospital, Taipei, Taiwan \\ ${ }^{\mathrm{b}}$ Center for Biomedical Engineering, College of Medicine, National Taiwan University, Taipei 10016, Taiwan
}

\begin{abstract}
The purpose of this study was to examine the relationship between tissue response and the membrane surface structure. A series of ethylene vinyl alcohol (EVAL) membranes provide an ideal system for a study of the relative contributions to biocompatibility derived from the surface morphology. This is different from other reports using different kinds of polymers, which may thus result in considerable variation since polymer implants are both chemically and physically heterogeneous. This study demonstrated that the tissue response to three different membrane structures were quite different. Flat and dense EVAL surfaces induced a mild foreign body reaction indicating that EVAL copolymer does not provide a stimulus for a continued inflammatory response. The inflammatory response was strong using a porous surface and a surface with a particulate morphology. In addition, particulate surfaces provoked a more intense inflammatory response than porous surfaces, indicating that a material in particulate form, suitable for phagocytosis, may provoke a different degree of inflammatory response than the same material in a nonphagocytosable form. Therefore, although a material may be considered to be biocompatible, however, its surface properties may alter the observed tissue reaction and inflammatory response. Consequently, the surface properties should be suitably selected according to the purpose for which the material is to be used. (C) 2000 Elsevier Science S.A. All rights reserved.
\end{abstract}

Keywords: EVAL membranes; Surface property; Inflammation; Complement activation

\section{Introduction}

Recently, research on polymers used for the construction of implants has increased considerably. To fulfill their functions, it is necessary that polymers possess good biocompatibility. Most biocompatibility tests are performed by inserting the biomaterials into the subcutaneous or muscle tissues. These implants initiate a complex set of biologic responses that determine and regulate local and systemic effects, such as inflammatory reactions [1-3] and complement activation [4]. The real situation in vivo is an extremely complex system, thus, the success of synthetic polymers as implants depends on many factors, such as implant chemistry, charge, size, shape and morphology.

Surface morphology is one of the factors influencing biocompatibility. White et al. [5] described the effects of

\footnotetext{
* Corresponding author. Tel.: +886-2-3970800 ext. 1455; fax: +8862-3940049.

E-mail address: thyoung@ha.mc.ntu.edu.tw (T.-H. Young).
}

pore size and material on soft tissue ingrowth of two medical-grade elastomers: silicone rubber and bioelectric polyurethane. Differences in the rate of tissue ingrowth were found to be related to both material and pore size. In addition, the inflammatory response against implanted porous biomaterials was more intense when compared to nonporous materials. Salthouse [6] showed that macrophage behavior was distinctly different at smooth and rough surfaces on polymers including polyamide, polyethylene terephthalate, polytetrafluoroethylene and polypropylene. At rough implant surfaces, macrophages were observed within $24 \mathrm{~h}$ and persisted for 3 months, while there were few cells even after 1 month of implantation using smooth surfaces. Similarly, Behling and Spector [7] compared the tissue compatibility of ultrahigh molecular weight polyethylene and polysulfone and indicated that rough surfaces contained higher percentages of foreign body giant cells, while smooth surfaces contained mainly fibrous tissue and macrophages. In a recent paper, Lam et al. [8] reported that biodegradable poly(L-lactic acid) films provoked a more 
intense inflammatory response than nondegradable polytetrafluoroethylene films and porosity also enhanced the inflammatory response. One problem derived from these papers using different kinds of polymers is that polymer implants are heterogeneous both chemically and physically, which may result in considerable variation. As a result, the complex in vivo environment makes it difficult to determine which factors cause an effect. We focused on the surface morphology of polymer implants in this study. Therefore, the implant materials had the same bulk chemistry but the surfaces were heterogeneous. We used the phase inversion method [9] to prepare ethylene vinyl alcohol (EVAL) membranes with different surface morphologies to perform tissue response tests. EVAL copolymer was chosen because it has been used in hemodialysis [10]. It is, therefore, possible to distinguish the effects of surface characteristics on biocompatibility from effects due to unknown factors. The phase inversion method is a simple and practical procedure to systematically control membrane surface structure, but does have a limitation in that polymer molecules may reorganize themselves so as to achieve the lowest possible interfacial tension during membrane formation. Hence, the surface region may be different in composition from the bulk. In this study, detailed surface characterization of EVAL membranes was done using electron spectroscopy for chemical analysis (ESCA). In addition, the effect of surface structure on complement activation was investigated using a specific and sensitive ELISA test for C5a.

\section{Experiment}

\subsection{Materials}

EVAL copolymer having a content of $56 \mathrm{~mol} \%$ of vinyl alcohol monomeric units was obtained using previously reported techniques [11]. De-ionized and ultrafiltrated water were used in our experiments. Other reagents were of chemical reagent grade and were used without further purification.

\subsection{Membrane preparation and characterization}

Membranes were prepared by the phase inversion method [9]. The EVAL copolymers were dissolved in dimethyl sulfoxide (DMSO) to form a $25 \%$ polymer solution at $60^{\circ} \mathrm{C}$. The solution was kept at $25^{\circ} \mathrm{C}$ for $24 \mathrm{~h}$ and was then spread on glass plates at a uniform thickness of $175 \mu \mathrm{m}$ at $25^{\circ} \mathrm{C}$ to prepare the membranes. Various EVAL membrane structures were fabricated by using the phase inversion method in our laboratory [12-14].

The present study concerns the following three membranes.

1. Membrane A: a dense and flat membrane was prepared by completely evaporating the DMSO at $70^{\circ} \mathrm{C}$.
2. Membrane B: a porous membrane was prepared by immersing the casting solution in water at $60^{\circ} \mathrm{C}$.

3. Membrane C: a membrane with a microparticulate morphology was prepared by immersing the casting solution in a DMSO/water mixture, containing 66\% DMSO at $25^{\circ} \mathrm{C}$.

The morphology of the membrane was examined using a scanning electron microscope (SEM). The freeze-dried samples were sputtered with gold and palladium in a vacuum and using a Hitachi S-800 microscope at $15 \mathrm{kV}$ for membrane A and $20 \mathrm{kV}$ for membranes $\mathrm{B}$ and $\mathrm{C}$, respectively. The surface chemical analyses of the membranes were carried out using ESCA. The ESCA spectra were obtained on an ESCA 850 photoelectron spectrophotometer (Shimadzu Seisakushyo) with a Magnesium anode $(\mathrm{MgK}=1253.6 \mathrm{eV})$ at $8 \mathrm{kV}$ and $30 \mathrm{~mA}$.

\subsection{Tissue response}

The aforementioned three membranes were cut into discs of $5 \mathrm{~mm}$ in diameter and sterilized by placing in alcohol. Prior to implantation, the membranes were washed with sterile saline solution. Male Wistar rats $(250-350 \mathrm{~g})$ were anaesthetized with diethyl ether and prepared for surgery by shaving their backs and then scrubbing with alcohol solution. The membranes were implanted subcutaneously in the back of the rat.

The animals were sacrificed periodically at 2 days, 1,2 , 4, 6, 8 and 16 weeks post-implantation. Three rats were employed for each time point. The implants including the surrounding tissue were excised and fixed in 10\% buffered formalin. Using the procedure of processing and embedding, the tissues were made into paraffin blocks. The tissues were then made into sections into 5-7 $\mu \mathrm{m}$ in thickness and stained with haematoxylin and eosin. After optical microscopic examination, photomicrographs of the stained sections were taken using a Leitz Orthoplan optical microscope (Germany) fitted with a micrographic attach-

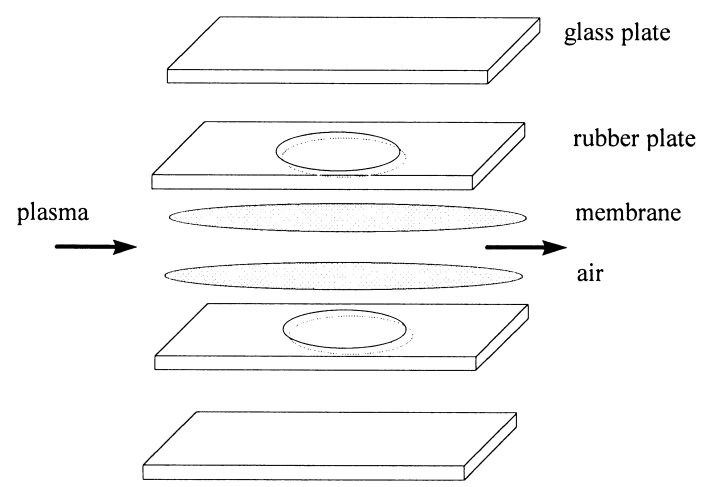

Fig. 1. Schematic illustration of complement activation in vitro by incubating different membranes in a sandwich. 
ment. This study received prior approval of the National Science Council of the Republic of China.

(a)

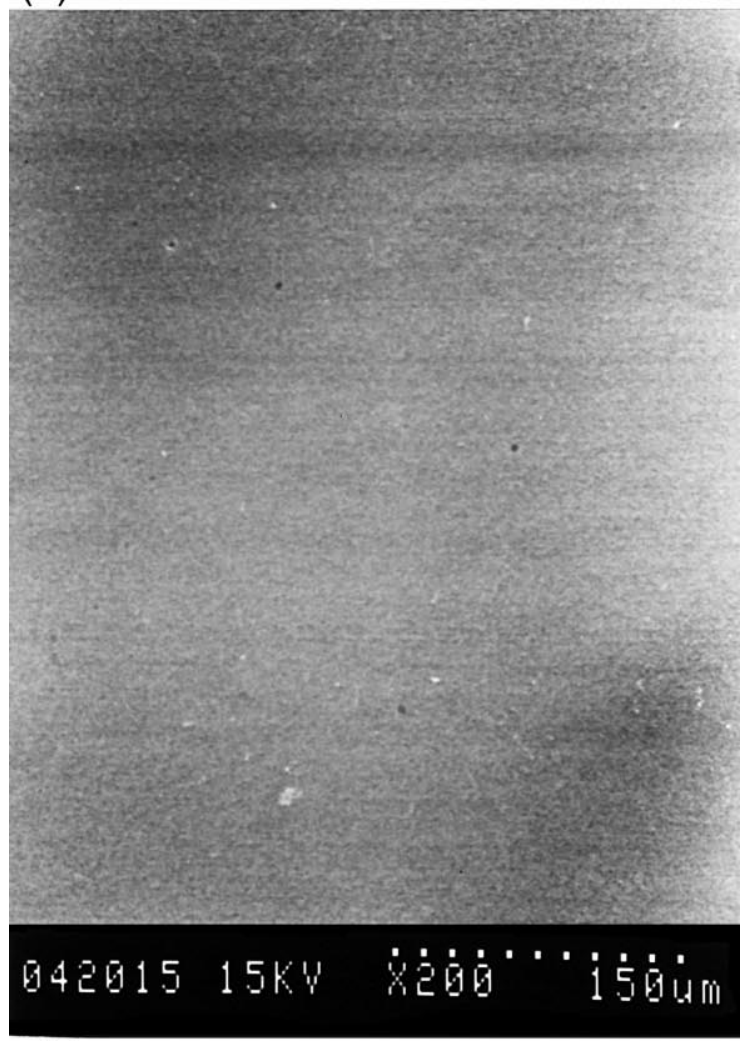

(b)

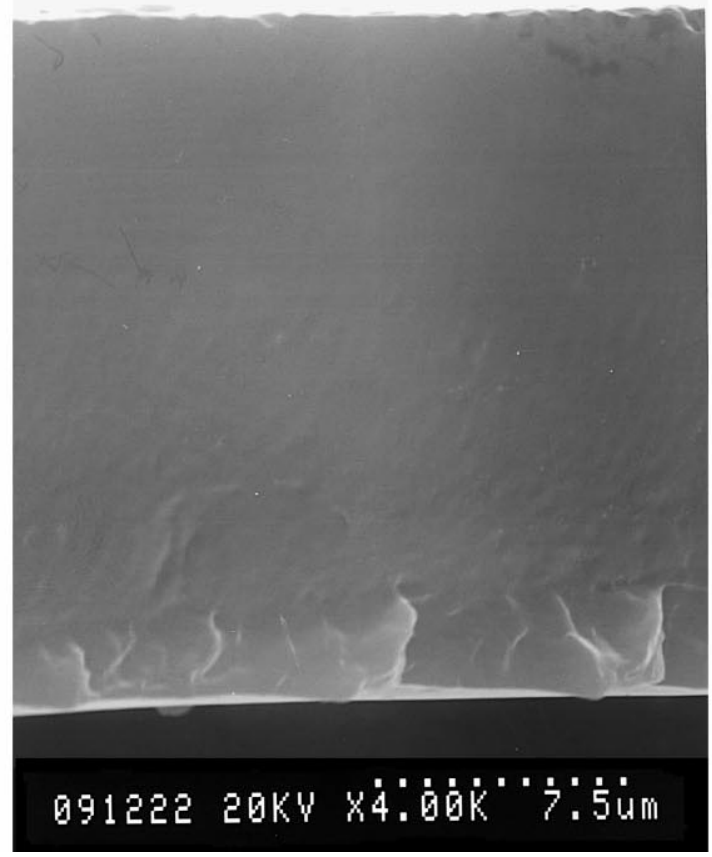

Fig. 2. SEM photomicrographs of EVAL membranes: (a) the top surface of membrane A, (b) the cross-section of membrane A, (c) the top surface of membrane B, (d) the cross-section of membrane B, (e) the top surface of membrane $\mathrm{C}$, and (f) the cross-section of membrane $\mathrm{C}$. (c)

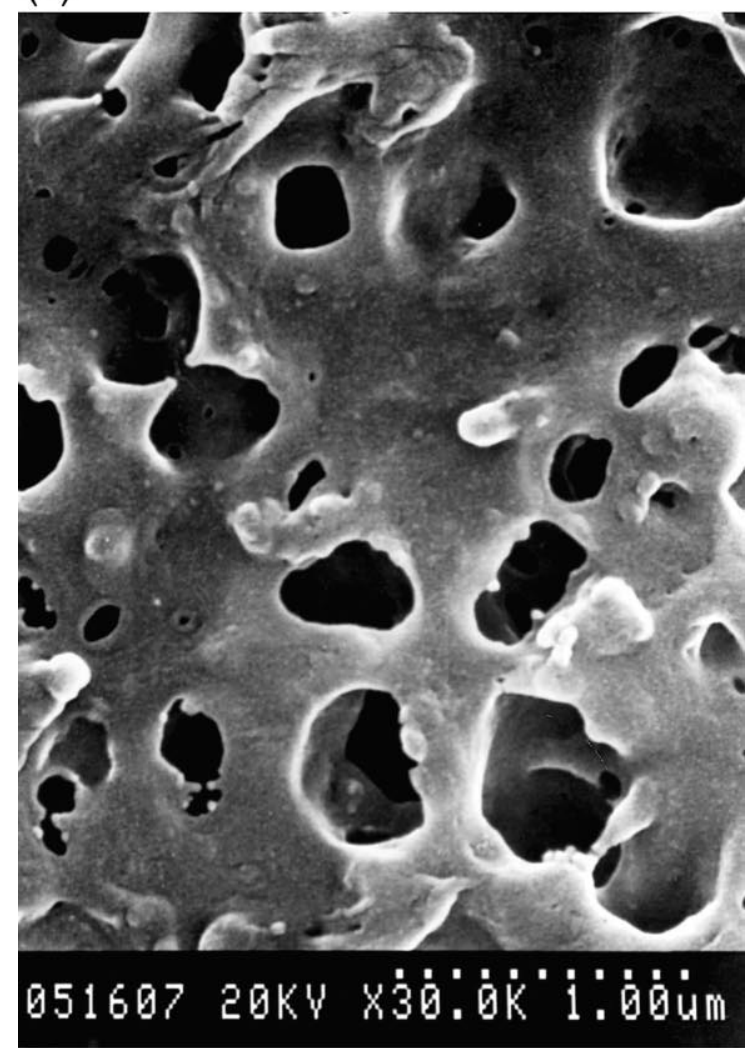

(d)

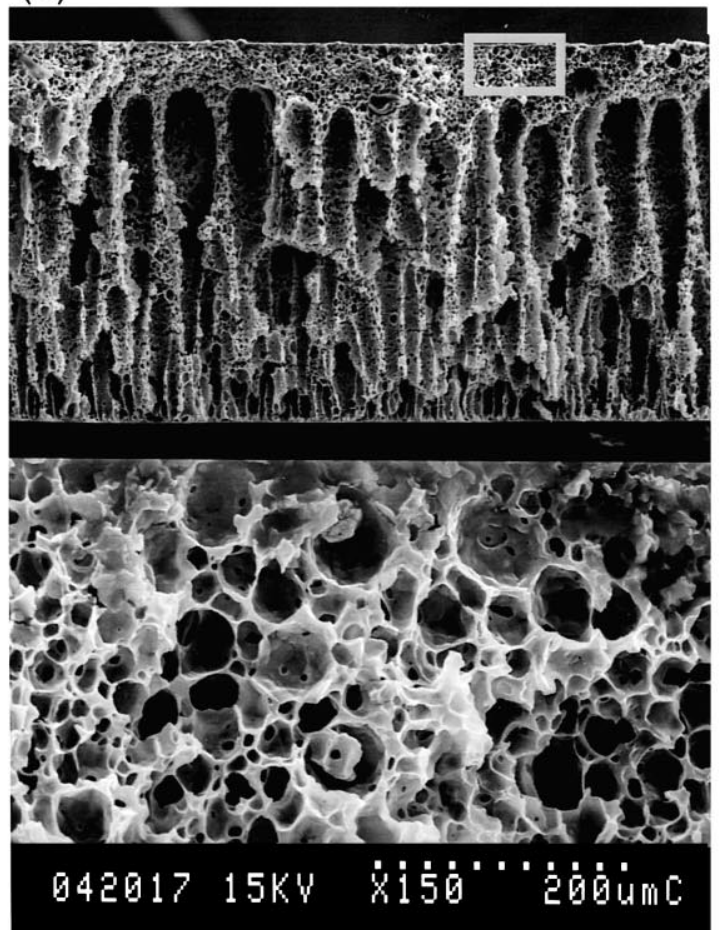

Fig. 2 (continued).

\subsection{Complement activation}

Blood from healthy donors was collected in vials containing citric acid $(0.5 \mathrm{mg}$ citric acid/ml blood) and 
(e)

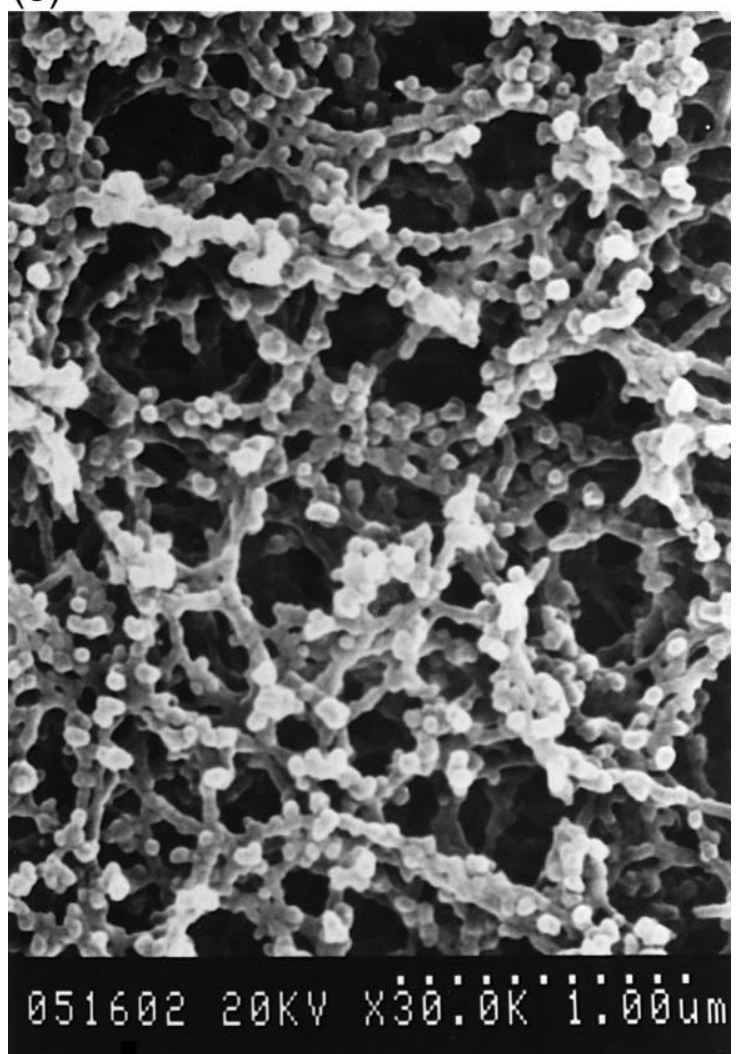

(f)

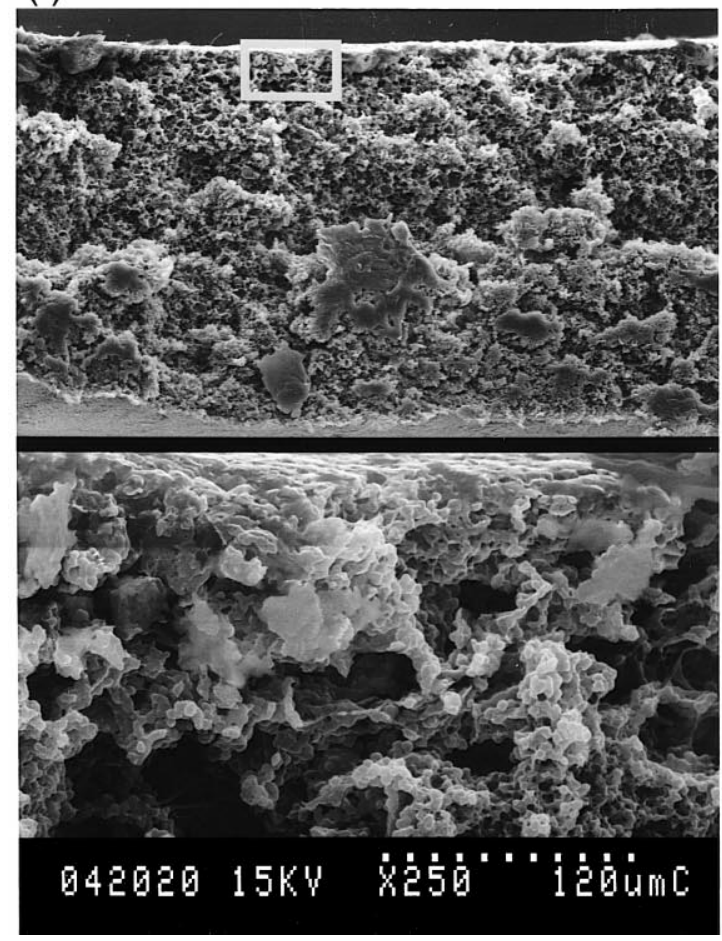

Fig. 2 (continued).

centrifuged immediately to obtain supernatant plasma. Complement activation was measured in vitro by incubating different membranes in a sandwich containing plasma on a rotating disk, as shown in Fig. 1. One milliliter of plasma was introduced with syringes into a thin channel between membranes. The membranes were positioned between two rubber plates, which have a pore in the center. Two pieces of glass were placed on the outside of the rubber plates and clamped. The total exposed area was about $6.3 \mathrm{~cm}^{2}$. During the injection of plasma, trapped air was removed by the needle on the other side. For each

(a)

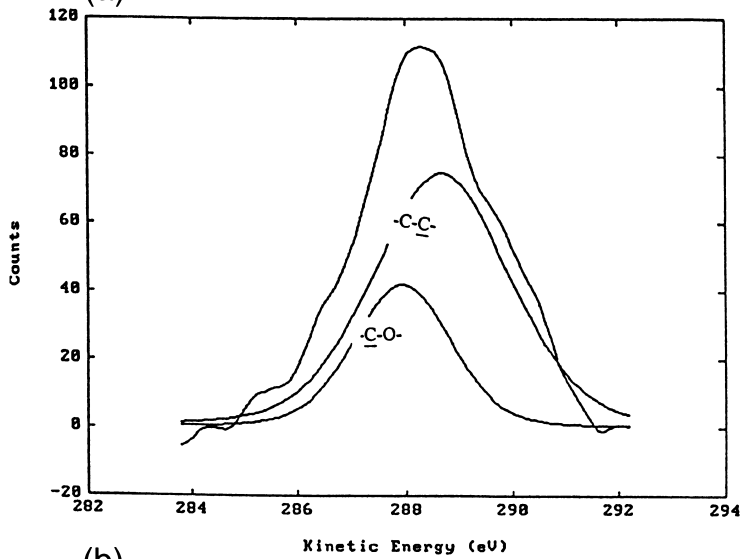

(b)

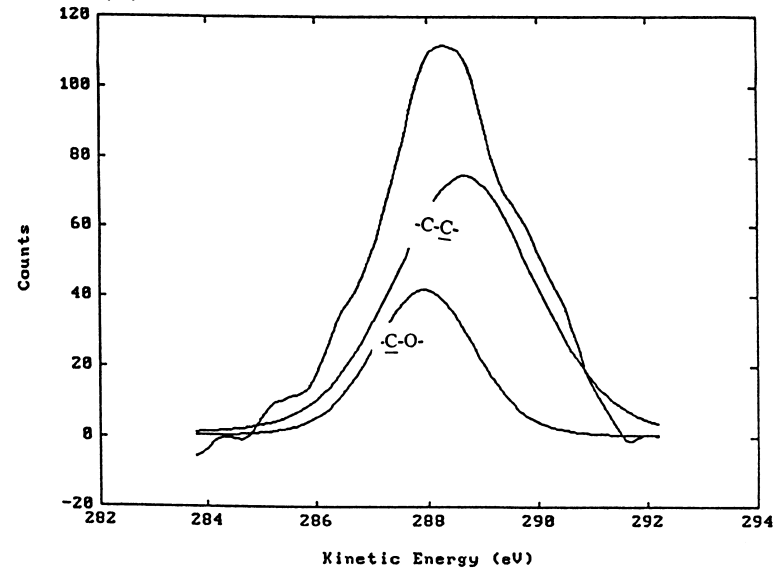

(c)

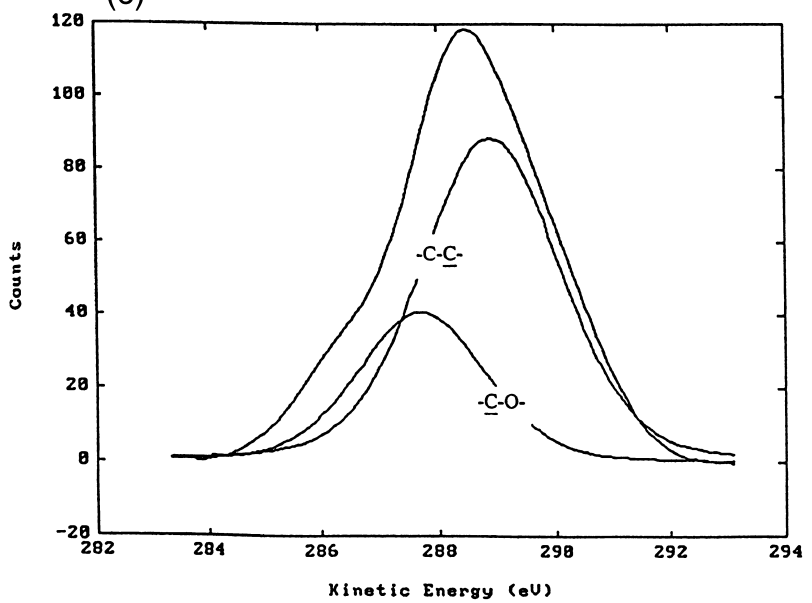

Fig. 3. Carbon 1 s core-level scan spectra of EVAL membranes (a) A, (b) $\mathrm{B}$, and (c) C. 
Table 1

ESCA analysis of the EVAL membrane surfaces

\begin{tabular}{llcl}
\hline Membrane & $-C-\mathrm{C}-{ }^{\mathrm{a}}$ & $-C-\mathrm{O}-^{\mathrm{a}}$ & $\mathrm{O} / \mathrm{C}$ ratio $^{\mathrm{b}}$ \\
\hline $\mathrm{A}$ & 382.9 & 72.38 & 15.9 \\
$\mathrm{~B}$ & 246 & 100.6 & 29.0 \\
$\mathrm{C}$ & 292.5 & 72.3 & 19.8 \\
\hline
\end{tabular}

${ }^{\mathrm{a}}$ The area under the carbon 1 score-level scan peak.

$\mathrm{b} \frac{\mathrm{O}}{\mathrm{C}}=\frac{A(-\mathrm{C}-\mathrm{O}-)}{A(-\mathrm{C}-\mathrm{O}-)+A(-\mathrm{C}-\mathrm{C}-)} 100 \% \quad ; A=$ Area.

time point, complement activation was evaluated by determining the C5a anaphylatoxin in the plasma. C5a concentration was determined using an ELISA method (Behringwerke, Marburg, Germany) as described [15]. The detection limit of the ELISA is calibrated to be $1 \mu \mathrm{g} / 1$ of C5a. In this study, C5a levels before the contacting of membranes with human plasma are below $1 \mu \mathrm{g} / \mathrm{ml}$. All determinations were carried out in duplicates. For each experimental value, four independent experiments were carried out. Data are expressed as mean \pm SEM (standard error of mean). Significance was assessed using Student's $t$-test for paired samples at the $p<0.05$ level of confidence.

\section{Results}

\subsection{Characterization of membranes}

In Fig. 2, the corresponding SEM views of the membranes are presented. Membrane A showed a fairly dense and smooth surface structure, and without any pores existing in the cross-section. The surfaces of both membranes B and $\mathrm{C}$ were found to be a porous structure, while the pore wall of membrane $\mathrm{C}$ revealed a high degree of particle connectedness of approximately submicron size. Since the structure of porous membranes $\mathrm{B}$ and $\mathrm{C}$ are asymmetric, all the tests in this study, including ESCA measurements, tissue response and complement activation, are specified in the membrane top surfaces (air-side surfaces).

The change in chemical structure of the EVAL membrane surface was investigated by ESCA. The EVAL membrane surface showed carbon (binding energy, ca. 288
$\mathrm{eV}$ ) and oxygen (binding energy, ca. $535 \mathrm{eV}$ ) peaks, as expected. Fig. 3 shows the carbon $1 \mathrm{~s}$ core-level scan spectra of EVAL membranes. The areas under the carbon $1 \mathrm{~s}$ core-level scans of the alkyl carbon $(-C-\mathrm{C}-)$ peak and carbon atoms with a hydroxyl group $(-\mathrm{C}-\mathrm{OH})$ peak are summarized in Table 1. The results indicate that the $\mathrm{O} / \mathrm{C}$ ratio of EVAL membranes was changed during membrane formation. Membrane B had the largest O/C ratio of 29.0. This reflects the fact that the hydroxyl group of membrane B may have migrated toward the surface at the moment the casting solution came into contact with water. The value of the $\mathrm{O} / \mathrm{C}$ ratio for membrane $\mathrm{C}$ was the smallest (15.9), when compared with that of membranes $B$ and $C$. This means that the hydroxyl groups were located at the subsurface or at a deeper region when membrane A was prepared by evaporating DMSO at $70^{\circ} \mathrm{C}$.

\subsection{Tissue response}

EVAL membranes were subcutaneously implanted in rats for up to 16 weeks and the short- and long-term tissue responses to these implants were studied. Macroscopically, there were no signs of infection demonstrated by any of the rats. Histological sections were studied chronologically. The changes of number of cells with implantation time were given in Table 2 on a semi-quantitative basis.

\subsubsection{Membrane A}

The sections taken from 2 days after implantation revealed granulation tissue formation admixed with an acute inflammatory response. One week after implantation, the inflammatory reaction shifted to a chronic response as evidenced by the presence of lymphocytes and fibroblasts. Fibroblastic proliferation surrounding the implant began to aggregate 2 weeks after implantation and subsequently form a thin fibrous capsule. No more progressive inflammatory reaction could be discerned after the formation of this capsule. At the end of 6 weeks, a connective tissue response was observed, indicating repair, equal to or greater than the inflammatory response for membrane A. After 8 weeks, fibroblasts became the main component of the surrounding cells (Fig. 4). From then on, except for the thin fibrous capsule, no other inflammatory response was

Table 2

Semi-quantitative analysis of the number of cells on EVAL membranes with implantation time

\begin{tabular}{|c|c|c|c|c|c|c|c|c|c|c|c|c|}
\hline \multirow[t]{3}{*}{ Cell } & \multicolumn{12}{|c|}{ Membrane } \\
\hline & $\bar{A}$ & B & $\mathrm{C}$ & A & B & $\mathrm{C}$ & $\mathrm{A}$ & B & $\mathrm{C}$ & $\mathrm{A}$ & B & $\mathrm{C}$ \\
\hline & \multicolumn{3}{|l|}{2 days } & \multicolumn{3}{|c|}{1 week } & \multicolumn{3}{|c|}{4 weeks } & \multicolumn{3}{|c|}{8 weeks } \\
\hline Lymphocyte & - & + & + & + & + & + & + & + & + & - & + & + \\
\hline Macrophage & + & + & + & - & + & + & - & + & + & - & + & + \\
\hline Fibroblast & - & + & - & ++ & + & + & ++ & + & + & ++ & ++ & + \\
\hline
\end{tabular}

$-:$ Item not present; $+:$ item present to a mild degree; ++ : item present to a marked degree. 


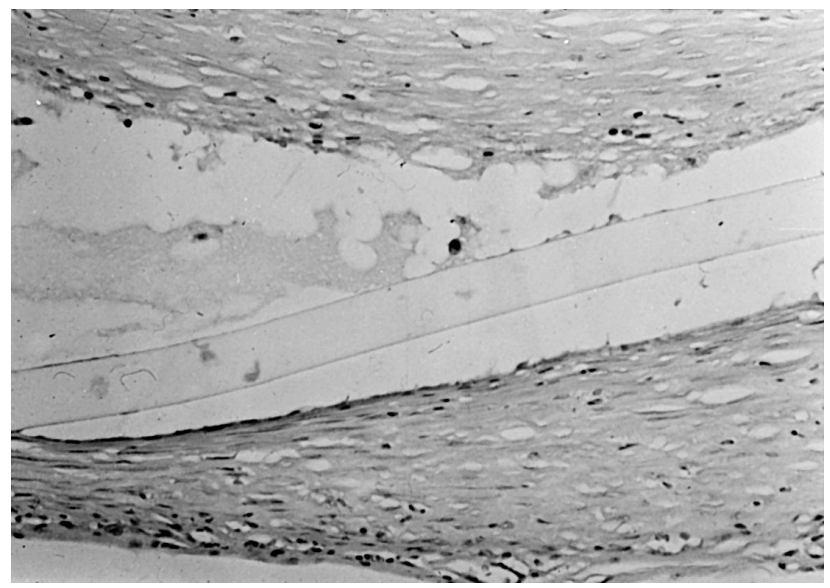

Fig. 4. After 8 weeks, fibroblasts have become the main component of the cells surrounding membrane A (original magnification $200 \times$ ).

observed. Even after 16 weeks, the fibrous capsule was maintained and no adverse tissue reactions were observed at the implant site. The inflammatory cells surrounding membrane A decreased rapidly with time, which is consistent with the situation expected for biocompatible polymers and indicates that EVAL copolymer as a dense structure does not provide a stimulus for a continued inflammatory response.

\subsubsection{Membrane B}

After 2 days of implantation of membrane $\mathrm{B}$, the immune reaction demonstrated invasion by scanty lymphocytes. This response was the same as that reported by Anderson [2]. Hence, this may be the subacute stage of the inflammatory reaction. The process of angiogenesis began in membrane B 1 week after implantation (Fig. 5a). In addition, the granulation tissue subjacent to the surface was shown to be composed of fibroblasts, macrophages, capillaries and collagen (Fig. 5b). After 4 weeks, increased amounts of connective tissue and the migration of macrophages towards the implant site were observed. This was followed by the invasion of foreign body giant cells formed as a result of the fusion of the macrophages at the end of 6 weeks. By post-implantation week 8 , these cells still persisted. Even at 16 weeks post-implantation, fibroblasts with scattered lymphocytes were observed around the membrane (Fig. 5c).

Summarizing from the tissue responses of membranes $\mathrm{A}$ and $\mathrm{B}$, it was found that the inflammatory cells surrounding membrane $\mathrm{B}$ were greater than for membrane A throughout the 16 weeks implantation period. This result is consistent with the report of Pans and Pierard [3], which stated that the difference in the behavior of the connective tissue is due to the different porosity of the material. The lower porosity of membrane A favored the process of cell accumulation on the borders of the biomaterial. The low porosity also determined the absence of blood vessels within membrane $\mathrm{A}$, in contrast to membrane $\mathrm{B}$, which, due to its greater porosity, underwent rapid angiogenesis.

\subsubsection{Membrane $C$}

The inflammatory reaction for membrane $\mathrm{C} 2$ days after implantation was more severe than that for membranes $\mathrm{A}$

(a)

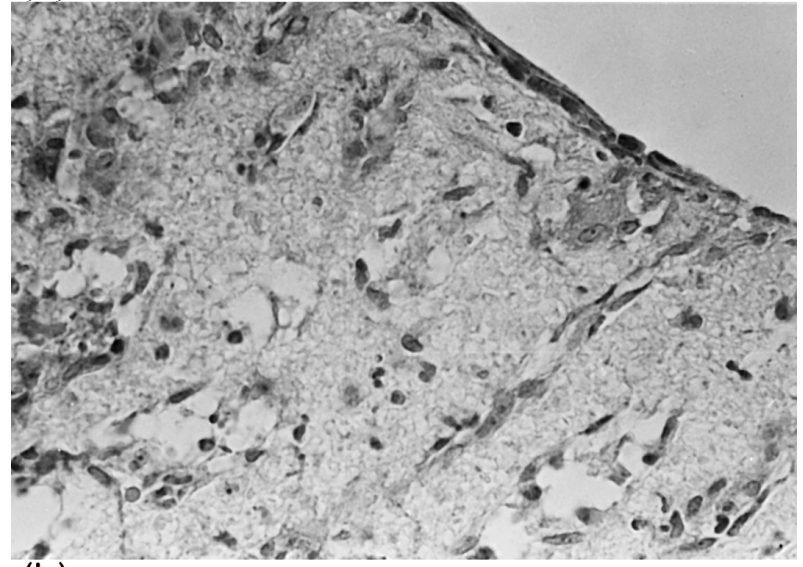

(b)

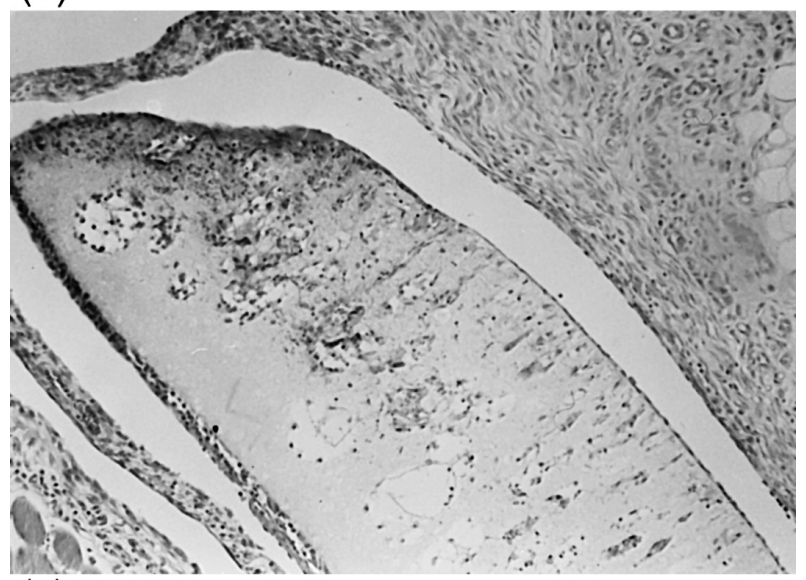

(c)

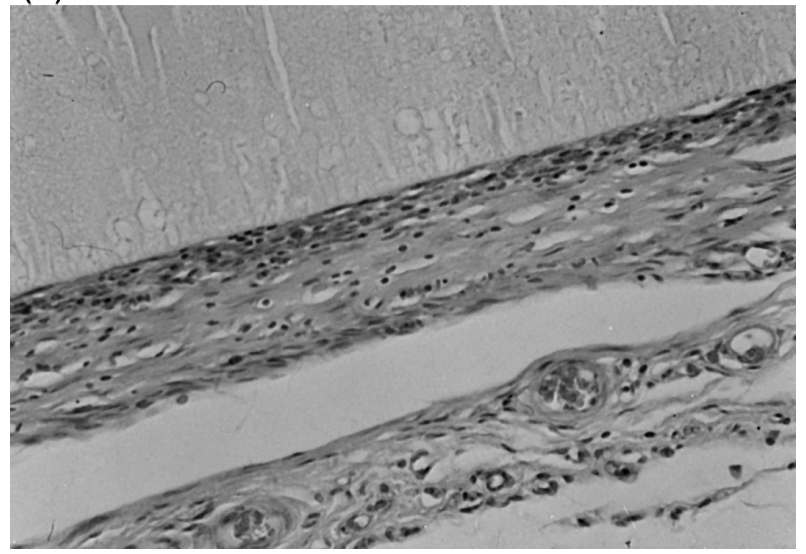

Fig. 5. (a) The process of angiogenesis has begun in membrane B 1 week after implantation (original magnification $400 \times$ ). (b) Granulation tissue subjacent to the surface of membrane B is composed of fibroblasts, macrophages, capillaries and collagen (original magnification $100 \times$ ). (c) At 16 weeks post-implantation, fibroblasts with scattered lymphocytes are observed around membrane B (original magnification $200 \times$ ). 
(a)

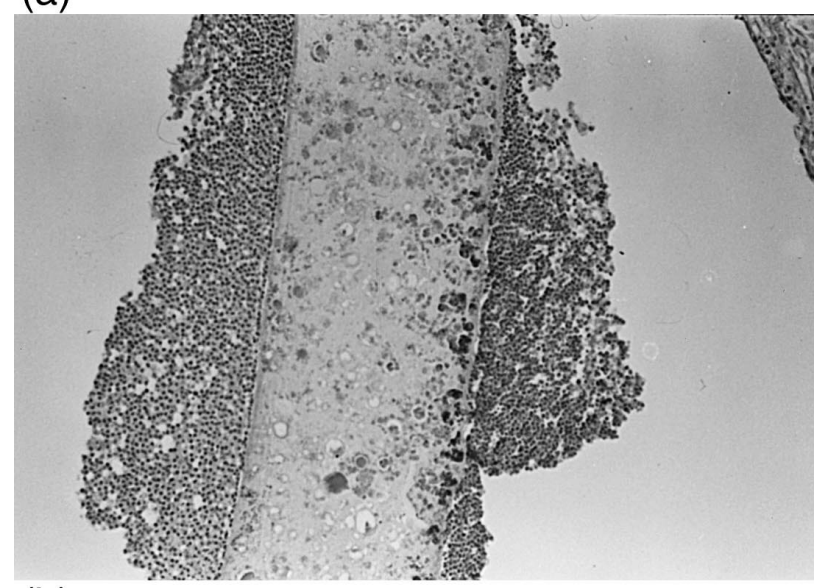

(b)

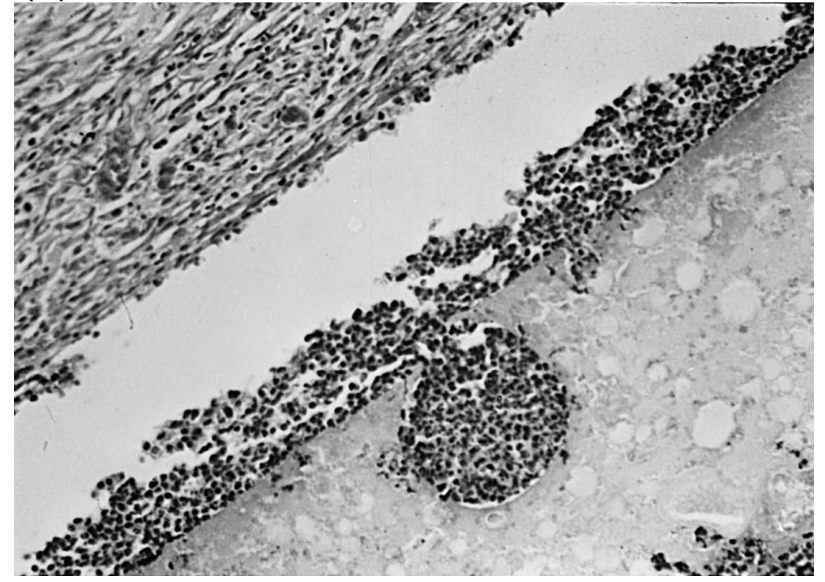

Fig. 6. (a) After 1 week, the density of the cells has increased and they have penetrated deeper into membrane $\mathrm{C}$ (original magnification $100 \times$ ). (b) At the end of 16 weeks, membrane $\mathrm{C}$ is enveloped by many cell layers and an abundant inflammatory cell reaction can be seen on the implant side (original magnification $200 \times$ ). and B. During the subacute inflammatory reaction, neutrophils, lymphocytes, and macrophages migrated into the wound site. The increased inflammatory response for membrane $\mathrm{C}$ could thus have been due to the particulate morphology. One important aspect was that inflammatory cells penetrated membrane $\mathrm{C}$ by day 2 post-implantation. After 1 week, the density of the cells increased and they penetrated deeper into the membrane (Fig. 6a). In addition, there always remained a portion with no cellular presence at some distance from the membrane. After 4 weeks, the distribution of inflammatory cells was fairly constant. At the end of 16 weeks, the membrane was enveloped by many cell layers and an abundant inflammatory cell reaction was found on the implant side (Fig. 6b). At this stage, phagocytosis of membrane $\mathrm{C}$ was visible. Membranes A and $\mathrm{B}$ showed no sign of phagocytosis, even after 16 weeks of implantation. The observed tissue reaction around membranes A and B had all the characteristics of classical fibrous encapsulation. Therefore, the greater degree of cellular reaction around membrane $\mathrm{C}$ has been due to its special particles-boned structure. The particle size was small enough to be phagocytized by macrophages [17], thus, degradation of the material could be expected, which could be responsible for the emergence of the neutrophils after 16 weeks of implantation.

\subsection{Complement activation}

EVAL membranes were incubated with the human plasma. At time points indicated in Fig. 7, plasma was analyzed for the content of C5a. The C5a concentration in the human plasma before contacting membranes was below the detection of the limit of the assay $(1 \mu \mathrm{g} / \mathrm{l})$. The measured C5a values for membranes B and C were corrected because porous membranes will contain more water

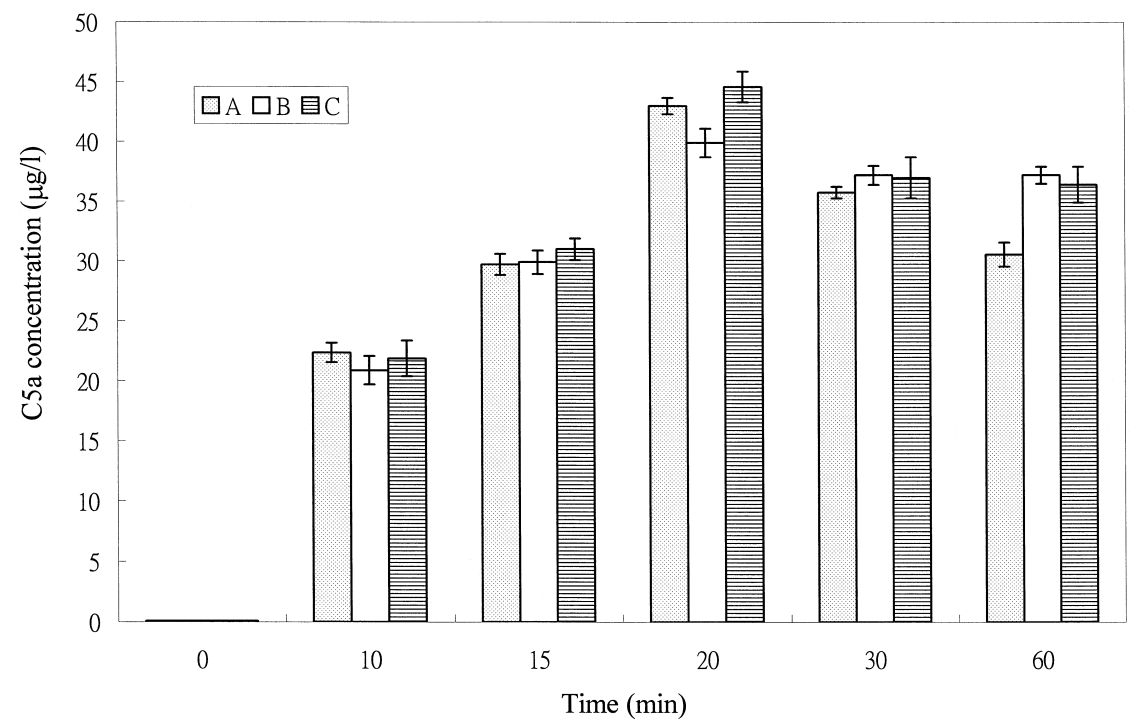

Fig. 7. C5a generation in human serum activated with EVAL membranes; $N=4$. 
to dilute the plasma. (The porosity of membranes B and C were 0.60 and 0.64 , respectively [18].) The plasma concentrations of C5a after 10 min of incubation were significantly elevated for all three membranes; see Fig. 7. Maximal complement activation occurred at $20 \mathrm{~min}$. At this time, the C5a level with different membranes did not differ significantly. Thereafter, the C5a level with membrane A appeared to express a faster decrease compared to those with membranes B and C. After $60 \mathrm{~min}$ of incubation, levels of C5a with membrane A were significantly lower than those with membrane $\mathrm{B}(p<0.05)$ or membrane $\mathrm{C}$ $(p<0.05)$, while these results for membranes B and C were not significantly different.

\section{Discussion}

The tissue response during the study period for all EVAL membranes can be seen to involve a mixture of both inflammatory neutrophils, macrophages, and lymphocytes migrating into the wound site and connective tissue. However, the results of histopathological examination represent the end stage of a repair process that begins when plasma proteins such as complement activation products contact and adhere to the material to induce a cellular response that may culminate in an inflammatory response [19]. For example, the alternative pathway of complement activation can occur through a transesterification mechanism between $\mathrm{C} 3 \mathrm{~b}$ molecules and $\mathrm{OH}$ or $\mathrm{NH}_{2}$ groups on the surface [4]. When bound, C3b is stabilized, then interacts with alternative pathway proteins to allow amplification of $\mathrm{C} 3$ activation and cleavage of $\mathrm{C} 5$ to generate $\mathrm{C} 5 \mathrm{a}$ in the presence of additional C3b. C5a is chemotactic for neutrophilic and eosinophilic granulocytes and for monocytes/macrophages [20,21].

To examine the possible existence of a relationship between the hydroxyl groups on membrane surfaces and complement activation, the induced C5a concentration on the membrane was measured. In our study, membrane A had the lowest $\mathrm{O} / \mathrm{C}$ ratio value. It agreed with the $\mathrm{C} 5 \mathrm{a}$ level with membrane A expressed a faster decrease from 20 to $60 \mathrm{~min}$ of incubation. On the other hand, there was a rapid reduction in the numbers of inflammatory cells between 1 and 8 weeks post-implantation, followed by a degree of stability persisting until week 16 of the study period. It appeared that the presence of hydroxyl groups on membranes is in some way related to the tissue response. However, membrane $\mathrm{B}$ had the largest $\mathrm{O} / \mathrm{C}$ ratio value, but the elevated plasma level of C5a incubated with the membrane $\mathrm{B}$ was not significantly larger than that of membranes $\mathrm{C}$ at all time points. Therefore, the generation of C5a by EVAL membranes may be effected by both the number of hydroxyl groups on its surface and the membrane surface morphology. (It is possible that microparticulate morphology is not beneficial to the complement activation. A thorough study of the effects of membrane surface morphologies on the complement activation will be followed in our laboratory.) In addition, membrane C provoked a more intense inflammatory response than membrane $\mathrm{B}$, indicating that a material in particulate form, suitable for phagocytosis, may provoke a different degree of inflammatory response than the same material in a nonphagocytosable form. Hence, the effect of the number of hydroxyl groups on the surface probably only resulted the limited correlation on the biocompatibility of EVAL membranes when their surface morphologies are so different on the system we studied. Consequently, membrane A with a relatively flat and dense surface induced a mild foreign body reaction. The inflammatory responses were substantially stronger for membranes B with a relatively porous surface and membrane $\mathrm{C}$ with a particulate morphology. Not only was the density of inflammatory cells in membranes $\mathrm{B}$ and $\mathrm{C}$ greater than that for membrane $\mathrm{A}$, but the inflammatory cells also invaded the membranes. Although, it would be naive to suggest that one parameter alone determines the tissue-implant interaction, obviously, it is difficult to compare the differences of biocompatibility of various implants regardless of the differences in their surface morphologies.

\section{Uncited reference}

\section{Acknowledgements}

The authors thank the National Science Council of the Republic of China for their financial support of project NSC 85-2331-B-002-181-M08.

\section{References}

[1] Y. Imai, E. Masuhara, Long term in-vivo studies of poly (2hydroxyethyl methacrylate), J. Biomed. Mater. Res. 16 (1982) 609617.

[2] J.M. Anderson, Inflammatory response to implants, Trans. Am. Soc. Artif. Intern. Organs 34 (1988) 101-107.

[3] A. Pans, G.E. Pierard, A comparison of intraperitoneal protheses for the repair of abdominal muscular wall defects in rats, Eur. Surg. Res. 24 (1992) 54-60.

[4] M.D. Kazatchkine, M.P. Carreno, Activation of the complement system at the interface between blood and artificial surfaces, Biomaterials 9 (1988) 30-35.

[5] R.A. White, F.M. Hirose, R.W. Sproat, R.S. Lawrence, R.J. Nelson, Histopathologic observation after short-term implantation of two porous elastomers in dogs, Biomaterials 2 (1981) 171-176.

[6] T.N. Salthouse, Some aspects of macrophage behavior at the implant interface, J. Biomed. Mater. Res. 18 (1984) 395-401.

[7] C.A. Behling, M. Spector, Quantitative characterization of cells at the interface of long term implants of selected polymers, J. Biomed. Mater. Res. 20 (1986) 653-666.

[8] K.H. Lam, J.M. Schakenraad, H. Groen, H. Esselbrugge, P.J. Dijkstra, J. Feijen, P. Nieuwenhuis, The influence of surface morphology 
and wettability on the inflammatory response against poly(L-lactic acid): a semi-quantitative study with monoclonal antibodies, J. Biomed. Mater. Res. 29 (1995) 929-942.

[9] R.E. Kesting, Synthetic Polymeric Membranes, Wiley, New York, 1985.

[10] Y. Sakurada, A. Sueoka, M. Kawahashi, Blood purification device using membranes derived from poly(vinyl alcohol) and copolymer of ethylene and vinyl alcohol, Polym. J. 19 (1987) 501-513.

[11] L.W. Chen, T.H. Young, EVAL membranes for blood dialysis, Makromol. Chem. Macromol. Symp. 33 (1990) 183-199.

[12] T.H. Young, L.W. Chen, Roles of bimolecular interaction and relative diffusion rate in membrane structure control, J. Membr. Sci. 83 (1993) 153-166.

[13] T.H. Young, L.W. Chen, Pore formation mechanism of membranes from phase inversion process, Desalination 103 (1995) 233-247.

[14] T.H. Young, L.W. Chen, L.P. Cheng, Membranes with a microparticulate morphology, Polymer 37 (1996) 1305-1310.

[15] M. Bingel, W. Arndt, M. Schulze, J. Floege, S. Shaldon, K.M. Koch, O. Gotze, Comparative study of C5a plasmal levels with different hemodialysis membranes using an enzyme-linked immunosorbent assay, Nephron 51 (1989) 320-324.
[16] J.M. Schakenraad, J.A. Oosterbaan, P. Nieuwenhuis, I. Molenaar, J. Olijslager, W. Potman, M.J.D. Eenink, J. Feijen, Biodegradable hollow fibers for the controlled release of drugs, Biomaterials 9 (1988) 116-120.

[17] S.M. Horowitz, S.B. Doty, J.M. Lane, A.H. Burstein, Studies of the mechanism by which the mechanical failure of polymethylmethacrylate leads to bone resorption, J. Bone Jt. Surg. 75-A (1993) 802-813.

[18] T.H. Young, C.H. Yao, J.S. Sun, C.P. Lai, L.W. Chen, The effect of morphology variety of EVAL membranes on the behavior of myoblasts in vitro Biomaterials, in press.

[19] W. Vogt, Anaphylatoxins: possible roles in disease, Complement 3 (1986) 177-188.

[20] R.O. Webster, S.R. Hong, R.B. Johnston Jr., P.M. Henson, Biological effects of the human complement fragments C5a and C5 $\mathrm{a}_{\text {des Arg }}$ on neutrophil function, Immunopharmacology 2 (1980) 201-219.

[21] A.B. Kay, H.S. Shin, K.F. Austen, Selective attraction of eosinophils and synergism between eosinophil chemotactic factor of anaphylaxis (ECF-A) and a fragment cleaved from the fifth component of complement (C5a), Immunology 24 (1973) 969-976. 